\title{
Electrical transport and EPR investigations: A comparative study for d.c. conduction mechanism in monovalent and multivalent ions doped polyaniline
}

\author{
SURESH KUMAR GUPTA*, VANDNA LUTHRA ${ }^{\dagger}$ and RAMADHAR SINGH \\ National Physical Laboratory (Council of Scientific and Industrial Research), Dr KS Krishnan Marg, \\ New Delhi 110 012, India \\ ${ }^{\dagger}$ Department of Physics, Gargi College, Siri Fort Road, New Delhi 110 049, India
}

MS received 26 May 2011; revised 8 November 2011

\begin{abstract}
A detailed comparative study of electron paramagnetic resonance (EPR) in conjunction with d.c. electrical conductivity has been undertaken to know about the charge transport mechanism in polyaniline (PANI) doped with monovalent and multivalent protonic acids. This work is in continuation of our previous work for further understanding the conduction mechanism in conducting polymers. The results reveal that the polarons and bipolarons are the main charge carriers formed during doping process and these cause increase in electrical conductivity not only by increase in their concentration but also because of their enhanced mobility due to increased inter-chain transport in polyaniline at high doping levels. EPR line asymmetry having Dysonian line shape for highly doped samples shows a marked deviation of amplitudes $A / B$ ratio from values close to one to much high values as usually observed in metals, thereby support the idea of high conductivity at higher doping levels. The nature of dopant ions and their doping levels control the charge carriers concentration as well as electrical conductivity of polyaniline. The electrical conductivity has also been studied as a function of temperature to know the thermally assisted transport process of these charge carriers at different doping levels which has been found to follow the Mott's variable range hopping (VRH) conduction model for all the three dopants used. The charge carriers show a change over from 3D VRH to quasi 1D VRH hopping process for multivalent ions at higher doping levels whereas 1D VRH has been followed by monovalent ion for full doping range. These studies collectively give evidence of inter-chain percolation at higher doping levels causing increase in effective mobility of the charge carriers which mainly seems to govern the electrical conduction behaviour in this system.
\end{abstract}

Keywords. Electron paramagnetic resonance (EPR); electrical conductivity; polyaniline; polaron-bipolaron; variable range hopping (VRH)

\section{Introduction}

Conducting polymers have been attracting considerable attention due to high electrical conductivity achievable by doping and their potential as highly tailorable semiconducting materials for their use in different technological applications including microelectronics (MacDiarmid et al 1987; Burroughes et al 1990; Lu et al 1995; Epstein 1997; Kulkarni et al 2005; Ayad et al 2010). Although the understanding of the mechanism of charge carriers motion in conducting polymers is of great importance, and has been studied in detail (MacDiarmid et al 1987; Singh et al 1997, 1998, 1999; Stejskal et al 2004; Krinichnyi et al 2006, Wu et al 2008), however, the generation of charge carriers with doping level and their relation with polymer structure and transport mechanism are still not completely understood. Polyaniline (PANI) is one of the most promising low cost conducting polymers having excellent thermal stability, which can be synthesized and doped very easily (MacDiarmid

\footnotetext{
*Author for correspondence (skgupta@mail.nplindia.org)
}

et al 1987; Stejskal et al 2004). It is distinguished from other conducting polymers because nitrogen heteroatoms are conjugated with other carbon atoms and the conjugated $\pi$-bonds are formed between $p_{\mathrm{z}}$-orbitals of carbon and nitrogen atoms of the polymer main chain. A variety of inorganic and organic dopants (Stejskal et al 2004; Singh et al 1997, 1998, 1999; Krinichnyi et al 2006) have been used with an aim to enhance and control its electrical conductivity. In the present work, electron paramagnetic resonance (EPR) spectroscopy (Alger 1968; Wilamowski et al 2011) and direct current (d.c.) electrical conductivity measurements have been undertaken for polyaniline doped with different inorganic acids viz. hydrochloric $(\mathrm{HCl})$, sulphuric $\left(\mathrm{H}_{2} \mathrm{SO}_{4}\right)$ and phosphoric $\left(\mathrm{H}_{3} \mathrm{PO}_{4}\right)$ acids. We have carried out this comparative study and correlated the EPR and d.c. conductivity data in continuation of our previous work (Rouleau et al 1995; Singh et al 1997, 1998, 1999; Luthra et al 2001, 2003) and efforts have been made to explain the charge conduction behaviour in polyaniline doped with monovalent and multivalent ions. Further the results of temperature dependence of d.c. electrical conductivity measurements have been analysed using 
Mott's variable range hopping (VRH) model (Mott and Davis 1979) to understand the thermally assisted transport process in this system.

\section{Experimental}

\subsection{Materials and methods}

Polyaniline is typically a phenylene based polymer consisting of reduced and oxidized repeat units as shown in figure 1 . When $y=0.5$, it consists of equal number of reduced and oxidized units and is known as emeraldine base (EB) form of polyaniline. When $y=1$, it is in fully reduced form called as leucoemeraldine and when $y=0$, it is in fully oxidized form and known as pernigraniline. EB form has been used in the present work since it is the most stable form among the three states. First, emeraldine salt (ES) form of polyaniline was synthesized by chemical oxidative polymerization of aniline in aqueous acidic medium $(1 \mathrm{M} \mathrm{HCl})$ using ammonium persulphate $\left[\left(\mathrm{NH}_{4}\right)_{2} \mathrm{~S}_{2} \mathrm{O}_{8}\right]$ as an oxidant (Chiang and MacDiarmid 1986; Singh et al 1997). This synthesized polyaniline in its protonated form (ES) was treated with $1 \mathrm{M}$ ammonium hydroxide $\left(\mathrm{NH}_{4} \mathrm{OH}\right)$ for $24 \mathrm{~h}$ which yielded emeraldine base (EB). EB powder was extracted with tetrahydrofuran in order to remove smaller polymer chains. Finally, EB powder was doped with $\mathrm{HCl}, \mathrm{H}_{2} \mathrm{SO}_{4}$ and $\mathrm{H}_{3} \mathrm{PO}_{4}$ acids by keeping $\mathrm{EB}$ powder in the aqueous solutions of various concentrations of these acids. These samples were washed with respective $\mathrm{pH}$ solutions of different acids followed by diethyl ether and acetone washings and were dried in vacuum for $24 \mathrm{~h}$. The polyaniline samples used in the present investigation have been designated as $A 1-A 5(\mathrm{HCl}$ doped), S1-S6 ( $\mathrm{H}_{2} \mathrm{SO}_{4}$ doped) and $P 1-P 6\left(\mathrm{H}_{3} \mathrm{PO}_{4}\right.$ doped $)$, respectively.

\subsection{Measurements}

EPR spectra of the samples were recorded on X-band EPR spectrometer (Varian Make, E-112) at $300 \mathrm{~K}$ (room temperature, $R T$ ) and $77 \mathrm{~K}$ (liquid nitrogen temperature, LNT). For these measurements, equal weights of powdered samples were filled in quartz capillary tubes having an internal diameter (ID) of $\sim 1 \mathrm{~mm}$. The particles in powdered samples of PANI were of size around $\sim 5 \mu \mathrm{m}$ as observed earlier in our electron microscopic investigations (Singh et al 1997, 1998). These capillary tubes were evacuated by using a diffusion pump (vacuum of the order of $10^{-6}$ Torr) and tubes

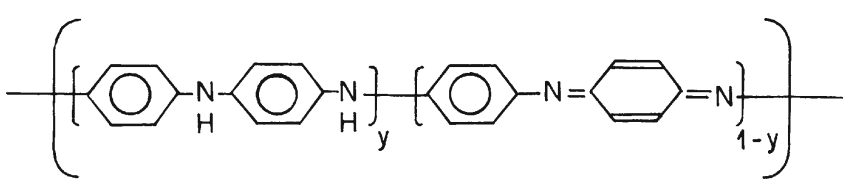

Figure 1. Structural formula of polyaniline. were sealed while keeping the vacuum system on. The sample tubes were placed in the centre of the rectangular EPR cavity having $\mathrm{TE}_{102}$ mode i.e. in the electric field node position where the microwave magnetic field has the maximum (Alger 1968). This excludes the chances of line asymmetry in EPR line shapes induced by electric component of the microwave field (Wilamowski et al 2011). D.C. magnetic field was modulated at $100 \mathrm{kHz}$ and modulation amplitude was kept very low $(0.05 \mathrm{G})$ to avoid any distortion in EPR line shape. Microwave power was also kept very low to avoid any saturation effect. DPPH was used as a standard reference sample (Hutchison and Pastor 1951; Feher and Kip 1955) for $g$-value determination and for the relative spin concentrations in different samples by comparing the integrated area of absorption curves of EPR signal with that of known amount of reference sample, DPPH. The same spectrometer settings, except different gain factors, were used for recording the spectra of all samples for determining spin concentration.

For d.c. conductivity measurements, powdered samples of PANI were pressed into pellet form (thickness, $\sim 1 \mathrm{~mm}$ ) and gold electrodes were deposited on both sides of pellets by thermal evaporation technique. The d.c. conductivity was measured by using two-probe method for lightly doped samples whereas four-probe method was used for highly doped samples using Keithley electrometer (model 617), constant current source (model 224) and nano voltmeter (model 181) in the temperature range $77-300 \mathrm{~K}$.

\section{Results and discussion}

A narrow single line EPR spectrum was obtained at $300 \mathrm{~K}$ $(R T)$ as well as at $77 \mathrm{~K}(L N T)$ for all samples of polyaniline similar to our earlier observation (Luthra et al 2003) indicating that the charge carriers have spin, $s=1 / 2$. As a representative result, EPR spectra of polyaniline doped with $\mathrm{H}_{3} \mathrm{PO}_{4}$ acid at different doping levels are shown in figure 2 . An increase in the intensity of EPR signal with increase in doping level has been observed for all the $\mathrm{HCl}, \mathrm{H}_{2} \mathrm{SO}_{4}$ and $\mathrm{H}_{3} \mathrm{PO}_{4}$ doped samples at both $R T$ and $L N T$. The EPR parameters calculated from these spectra are given in table 1. Some room temperature EPR data for $\mathrm{H}_{2} \mathrm{SO}_{4}$ doped samples have been included in this table from our previous work (Luthra et al 2003) for more comprehensive analysis of the results. At room temperature, the peak to peak line width of EPR signal has been found to vary from 6.0 to $3.7 \mathrm{G}$ and effect of narrowing of line width with increase in doping level has been observed in all the three categories of samples (table 1). The $g$-values have remained in the range of 2.0036 to 2.0042 i.e. close to free electron $g$-value (2.0023), which suggests that the resonance comes from the electrons delocalized in the $\pi$-bonded system of these conjugated polymeric systems consisting of $p_{\mathrm{z}}$ orbitals of carbon and nitrogen atoms in the main chain of polyaniline. On comparing with theoretical line contours, the observed line shapes of EPR signals of all the samples having low and moderate doping levels have been found to be of Lorentzian line shape. At high doping 


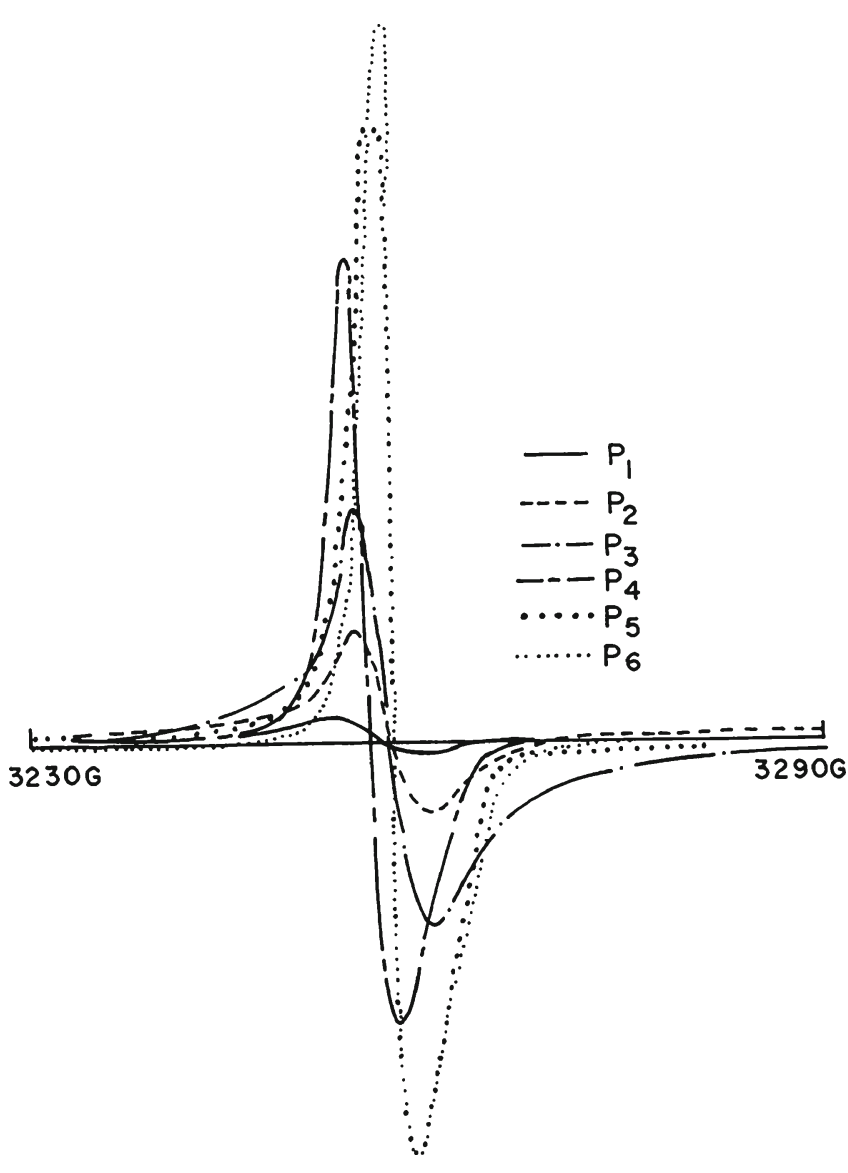

Figure 2. Relative variation of EPR signal intensity for $\mathrm{H}_{3} \mathrm{PO}_{4}$ doped polyaniline samples at $300 \mathrm{~K}$.

levels, Dysonian asymmetry in line shape characterized by high ratio of $A / B$, low field peak to high peak field amplitudes is obtained as usually observed in metallic samples (Alger 1968). It is worthwhile to mention here that in conducting samples due to the finite penetration of microwave field in the material, known as skin effect (Stephen and Frederic 1985), an asymmetric EPR signal of Dysonian line shape is obtained due to the admixture of absorption and dispersion components of a symmetric Lorentzian line shape (Dyson 1955; Alger 1968; Joshi and Bhatt 2004; Wilamowski et al 2011). Dyson (1955) has shown that when the skin depth becomes smaller than the sample thickness, the asymmetric line shape is attributed to the presence of a dispersive component in the line shape. In such cases, an additional line shape parameter $A / B$ ratio describes the fraction of the dispersion added on to the absorption and is required to describe the asymmetry of the signal. In polyaniline fully doped with $\mathrm{HCl}$ which is highly conducting, the skin depth of microwave at around $9.5 \mathrm{GHZ}$ is of the order of $1 \mu \mathrm{m}$ (Chipara et al 2004). Since the samples studied here did not have relatively such a high conductivity, skin depth was expected to be more than $1 \mu \mathrm{m}$.

On the basis of $g$-values, line width, line shape behaviour and earlier observations by other researchers (Lux 1994; Luthra et al 2003; Krinichnyi et al 2006), the EPR signal obtained has been assigned due to polarons (Bredas and Street 1985) formed during doping. When an electron is added to the polymeric chain it polarizes the surrounding media, a potential well is formed and added electron gets trapped in it. Polaron thus trapped electron in its distorted lattice. Both defect and trapped electron move together during electrical conduction. These polarons have a spin, $s=1 / 2$, give single line EPR signal and take part in electrical conduction phenomenon. The relative spin concentration of these charge carriers has been determined and found to increase with increase in the doping level (table 1). This increase in charge carrier concentration results into enhanced electrical conductivity of the doped samples.

It may be noted that for an arbitrary EPR line shape, the evaluation of paramagnetic centres concentration based on EPR spectrum becomes more complex (Feher and Kip 1955; Alger 1968). In conducting samples when Dysonian line shape is obtained, only a fraction of the sample spin absorbs the microwave power and therefore, EPR signal intensity does not reflect the total number of spin concentration present in the sample. In such cases, absolute value of spins may be determined experimentally by taking thin films or powder samples having thickness smaller than the skin depth. Since size of the particles studied here is more as compared to the skin depth, therefore, spin concentration of the charge carriers determined (table 1) represents only the trend of the variation of the relative spin concentrations in these samples at different doping levels. In fact, the exact values of spin concentrations are not required in the discussion presented here. The variation of relative spin concentration of charge carrier viz. polarons vs $\mathrm{pH}$ (doping level), is given in figure 3 which shows the tendency to saturate at higher levels of doping for all three kinds of dopants. The concentration of these charge carriers formed has also been found to depend upon the nature of the dopant ions used. For monovalent ions, more charge carriers have been formed compared to divalent or trivalent ions at low and moderate doping levels. A typical increase in spin concentration has been observed which is of the order of $10^{18}$ to $10^{20}$ spins/g i.e. spin concentration has increased by about 2 orders of magnitude. The change in concentration of polarons and effect of oxygen exposure on it in $\mathrm{HCl}$ doped polyaniline and in microporous polyaniline samples have also been reported earlier (Kang et al 1999; Langer et al 2001).

The variation in d.c. conductivity vs $\mathrm{pH}$ (doping level) is shown in figure 4 . These curves have been redrawn by including some data from our previous work (Singh et al 1997; Luthra et al 2003) for comparison of conductivity of different dopants. This figure indicates that the d.c. conductivity has increased with the increase in doping level for all dopants. The room temperature d.c. conductivity of all these doped polyaniline samples (table 1) shows a change of about 9 order of magnitude for $\mathrm{HCl}$ doped samples and about 7 orders of magnitude for $\mathrm{H}_{2} \mathrm{SO}_{4}$ and $\mathrm{H}_{3} \mathrm{PO}_{4}$ doped samples as doping level is increased from base form $\mathrm{EB}(\mathrm{pH} \geq 7)$ to the salt form $\mathrm{ES}(\mathrm{pH} \sim 0 \cdot 2)$ of polyaniline. It may also be observed here that the magnitude of conductivity at the same $\mathrm{pH}$ value 


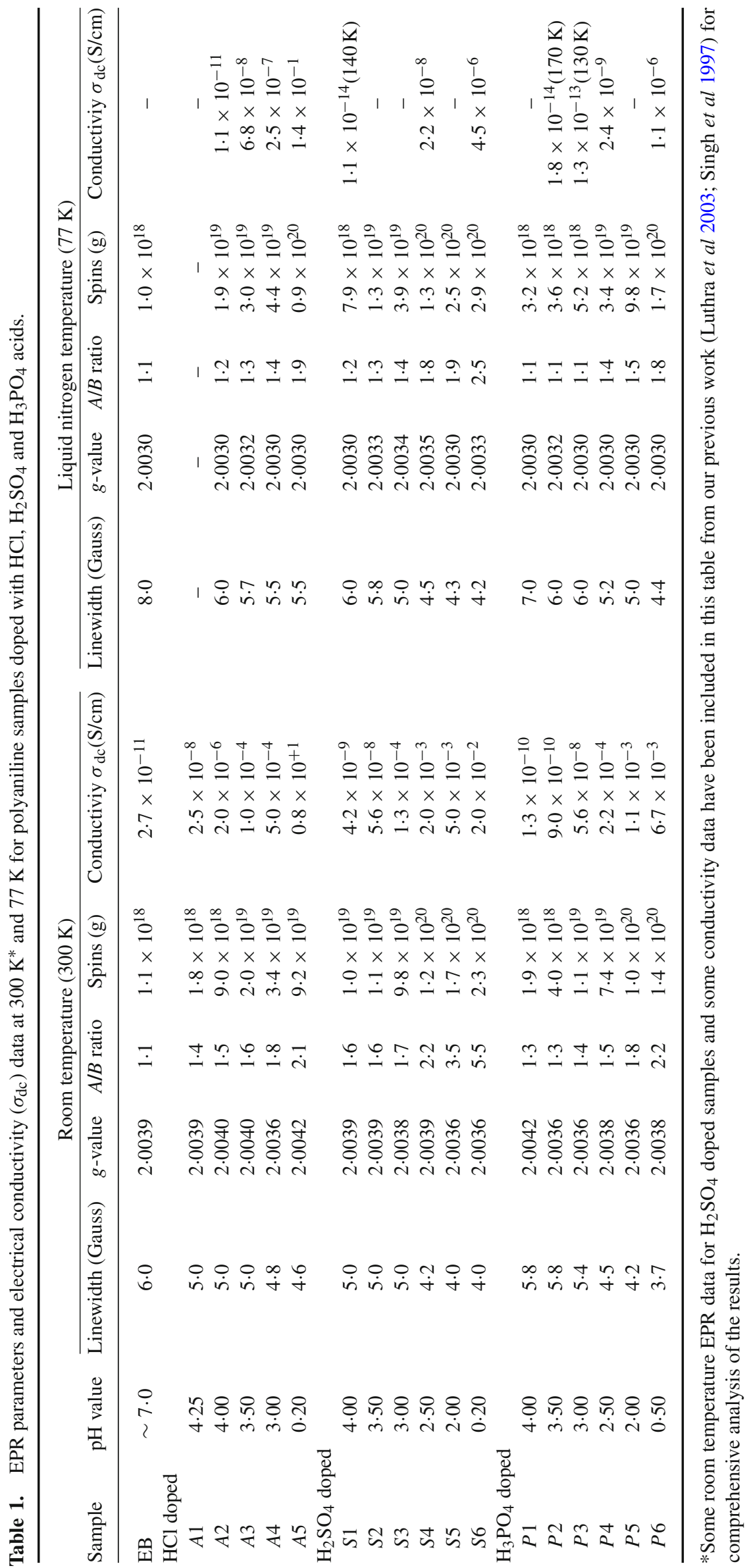


has been decreased from $\mathrm{HCl} \rightarrow \mathrm{H}_{2} \mathrm{SO}_{4} \rightarrow \mathrm{H}_{3} \mathrm{PO}_{4}$ acid used for protonation which suggests that the conductivity depends upon the nature of dopant and in the present case, it remains maximum for monovalent ion i.e. $\mathrm{HCl}$ dopant.

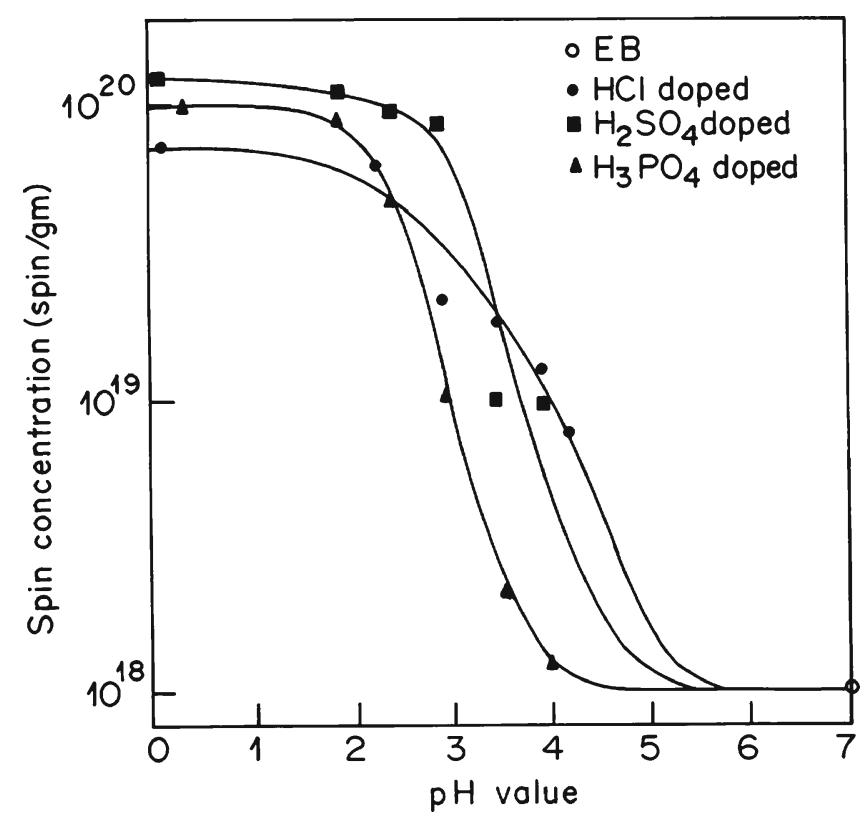

Figure 3. Variation of spin concentration in doped polyaniline samples at different $\mathrm{pH}$ levels.

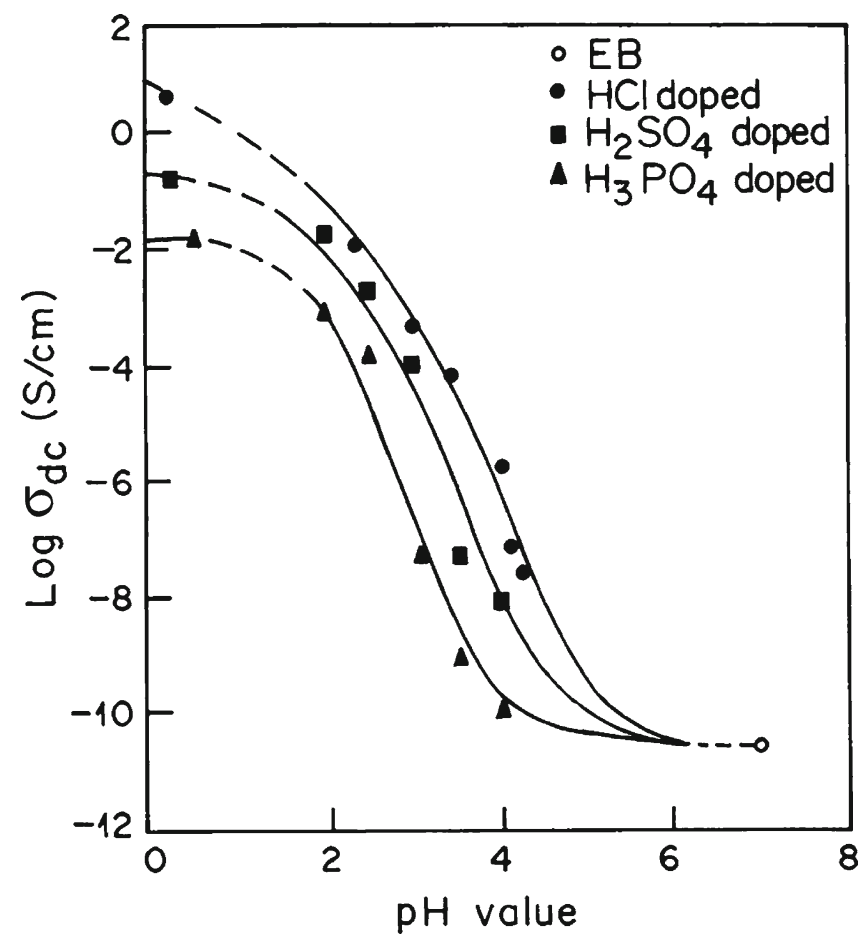

Figure 4. Variation of room temperature d.c. conductivity at different $\mathrm{pH}$ levels for doped polyaniline samples.
A correlation of EPR and d.c. conductivity data shows that at low and moderate doping levels, increase in spin concentration indicates increase in the polarons formation which carry electric charge and have a spin, $s=1 / 2$, thereby responsible for the increase in EPR signal intensity as well as electrical conductivity with an increase in doping levels. It may be noted here that the conductivity has increased more rapidly as compared to increase in spin concentration particularly at higher doping levels (table 1). As mentioned earlier, for full doping range, there is a change of about 9-7 orders of magnitude in electrical conductivity, whereas the spin concentration has increased only by two orders of magnitude. It suggests that the increase in electrical conductivity is not only due to increase in charge carriers concentration but apart from other factors, it may also be due to enhanced charge carrier mobility at higher doping levels.

In fact development of quantitative model for electrical conduction phenomenon in conducting polymers is hampered by the fact that there are at least three factors contributing to the charge carriers mobility viz. single chain or intra-molecular transport, inter-chain or between chain transport and inter-particle contact (Formmer and Chance 1986). These three factors make the resistive network complicated which determines the effective mobility $(\mu)$ of the charge carriers. Thus the mobility and therefore, the electrical conductivity is determined both at microscopic (intra- and interchain) and macroscopic (inter-particle) levels. For $\mathrm{HCl}$ doped polyaniline samples we have earlier observed in our structural electron microscopic studies carried out as a function of doping level that two separate phases are observed on increasing the doping level (Singh et al 1997, 1998). This observation is consistent with the fact that the protonation gives rise to segregation into fully protonated conducting phases embedded in an unprotonated matrix. This suggests that at low doping levels, the polymer chains are isolated from each other resulting in a highly anisotropic behaviour and at higher doping levels when the protonated chains begin to percolate, the inter-chain transport increases dramatically making carriers more mobile (Mizoguchi et al 1989). Thus in the present work, increase in electrical conductivity is realized both in terms of increase in carrier concentration and increased carrier mobility due to increased inter chain transport (Mizoguchi et al 1989; Lux 1994; Luthra et al 2003) at higher doping levels. Further it may be noted here that in the case of mobile spins, as in conducting polymers, the motion characteristics of charge carriers such as their mobility and dimensionality also play a major role in determining the EPR line shape and line width (Houze et al 1997). For highly doped polymer samples, line width of EPR signal has reduced considerably (table 1) due to well known process of motional narrowing which confirms fast motion of spin carriers (Elliott 1954; Alger 1968; Houze et al 1997) indicating thereby high mobility of the charge carriers at high doping levels. EPR line asymmetry has also been found higher for these highly doped samples having Dysonian line shape whose asymmetry parameter $(A / B)$ is related to the electrical conductivity of materials (Elliott 1954; Dyson 1955; 
Alger 1968). Hence, EPR observations confirm the idea of high mobility and high conductivity at higher doping levels in conducting polymers.

On further increasing the doping levels i.e. at very high doping levels, a further increase in spin concentration of charge carriers has not been observed and it tends to saturate (figure 3) though there is still an increase in electrical conductivity (figure 4). These observations suggest that there may be formation of other types of charge carriers like spinless bipolarons at such higher doping levels (Scott et al 1983; de Oliveria and dos Santos 2000; Luthra et al 2003). Bipolarons are doubly charged and have zero spin and therefore, can contribute only in electrical conduction but do not give EPR signal. This makes EPR unable to count these bipolaron carriers thereby charge carriers concentration seems to saturate at such high doping levels. On the basis of semiempirical quantum chemical calculations (de Oliveria and dos Santos 2000), it has been shown that bipolaron creation energy exceeds that of the creation of a pair of polarons but it may co-exist with polarons in polyaniline. Thus the d.c. conductivity data and EPR observations provide support in favour of polaron formation at low and moderate doping levels whereas at higher doping levels, where inter-chain percolation of the lattice takes place, bipolarons formation or polaron-bipolaron equilibrium is more favourable.

Detailed EPR measurements have also been made at $77 \mathrm{~K}$ to know the effect of cooling on the charge carriers concentration and their mobility because a decrease in electrical conductivity by $2-6$ orders of magnitude has been observed on cooling the sample from $300 \mathrm{~K}$ down to $77 \mathrm{~K}$ (see table 1). The $g$-values (2.0030-2.0035) and line width $(8.0 \mathrm{G}-4.2 \mathrm{G})$ of EPR lines obtained at $77 \mathrm{~K}$ have been found to remain in the same range as at $300 \mathrm{~K}$ given earlier. The appreciable change in relative spin concentration has also not been observed at $77 \mathrm{~K}$. However, Dysonian asymmetry $A / B$ ratio of EPR lines has been found to decrease significantly at $77 \mathrm{~K}$ (table 1) for all samples indicating thereby that charge carriers have become relatively slow at low temperature. Therefore, the study of temperature dependence of d.c. conductivity has been undertaken in the temperature range $77 \mathrm{~K}$ to $300 \mathrm{~K}$ to understand the thermally activated transport process.

For all the doped polyaniline samples, resistivity vs temperature measurement has shown that the room temperature electrical conductivity has increased with increase in temperature. This gives the indication that the underlying conduction mechanism is due to hopping of charge carriers typical of a semiconductor (Mott and Davis 1979). Conducting polymers are predominantly amorphous in nature and have substantial amount of disorder. Therefore, narrow band tails of localized states of valence and conduction bands and a narrow band of localized levels in mid of the energy gap are formed in conducting polymers (Mott and Davis 1979). At very low temperature, conduction may occur by thermally assisted tunneling between states at Fermi level but at higher temperature, charge transport mainly takes place due to hopping of the charge carriers excited in localized states of band tails. Therefore, the results of present investigation have been analysed in terms of Mott's variable range hopping (VRH) conduction model (Mott and Davis 1979) in which temperature dependence of d.c. conductivity is given by

$$
\sigma_{\mathrm{dc}}(T)=\sigma_{0} \exp \left(-T_{0} / T\right)^{m},
$$

where $\sigma_{0}$ and $T_{0}$ are constants, $m=1 /(d+1)$ and $d$ the dimensionality of the charge hopping process, which makes $m=1 / 2$ for one dimensional (1D), $m=1 / 3$ for two dimensional (2D) and $m=1 / 4$ for three dimensional (3D) hopping transport. It is worthwhile to mention here that the Mott's VRH model has been extensively applied to various amorphous inorganic semiconductors (Mott and Davis 1979) over the last two decades and has also been applied with varying degrees of success to organic conducting polymers (Punkka et al 1991; Singh et al 1996, 2006a, b). In our case, as a representative result, the variation of d.c. conductivity $\left(\sigma_{\mathrm{dc}}\right)$ for $\mathrm{H}_{2} \mathrm{SO}_{4}$ doped samples ( $\left.S 1, S 2, S 4, S 6\right)$ and $\mathrm{H}_{3} \mathrm{PO}_{4}$ doped samples $(P 2, P 3, P 4, P 6)$ have been shown as functions of $T^{-1 / 2}$ and $T^{-1 / 4}$ in figures 5 and 6 , respectively. The conductivity curves for $P 2$ and $P 3$ samples have been included

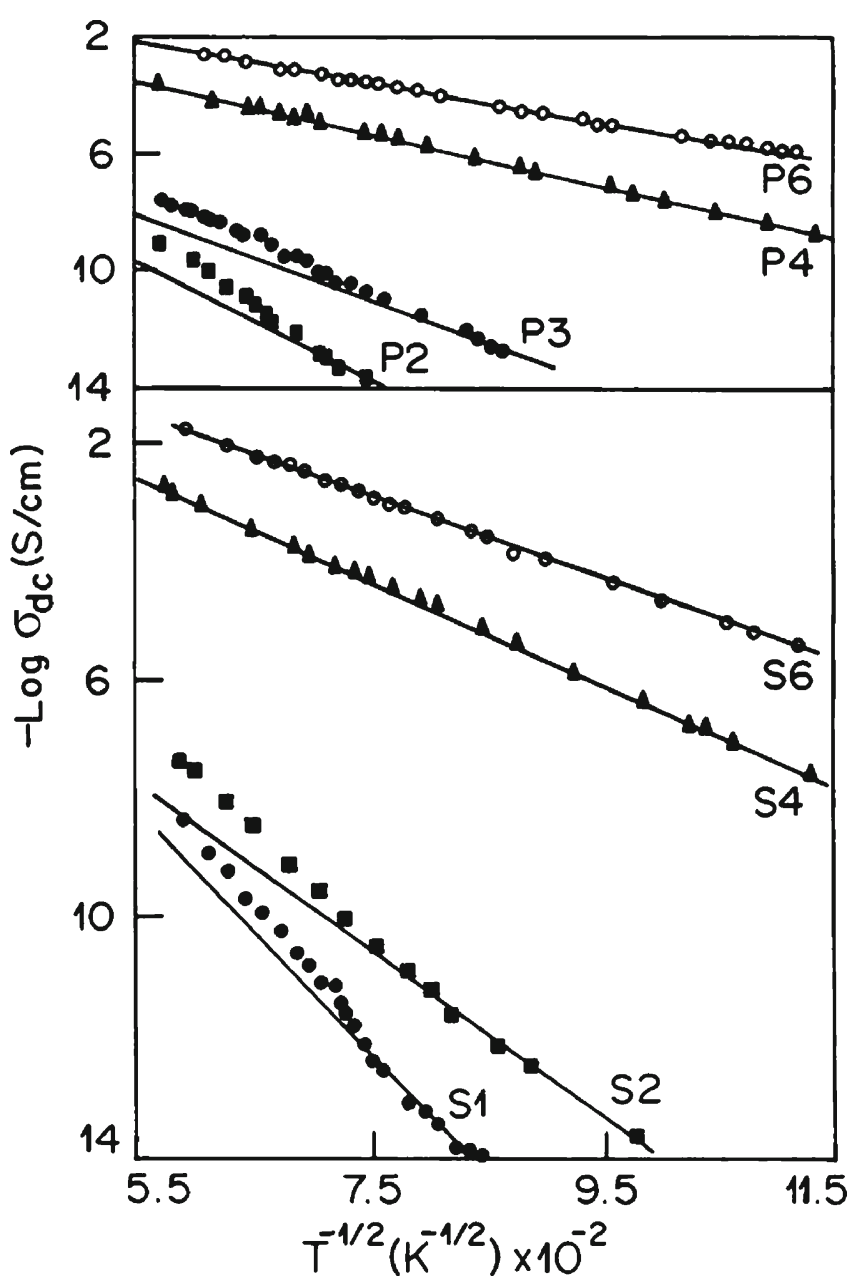

Figure 5. Variation of d.c. conductivity as a function of $T^{-1 / 2}$ for different $\mathrm{H}_{2} \mathrm{SO}_{4}$ and $\mathrm{H}_{3} \mathrm{PO}_{4}$ doped polyaniline samples. 


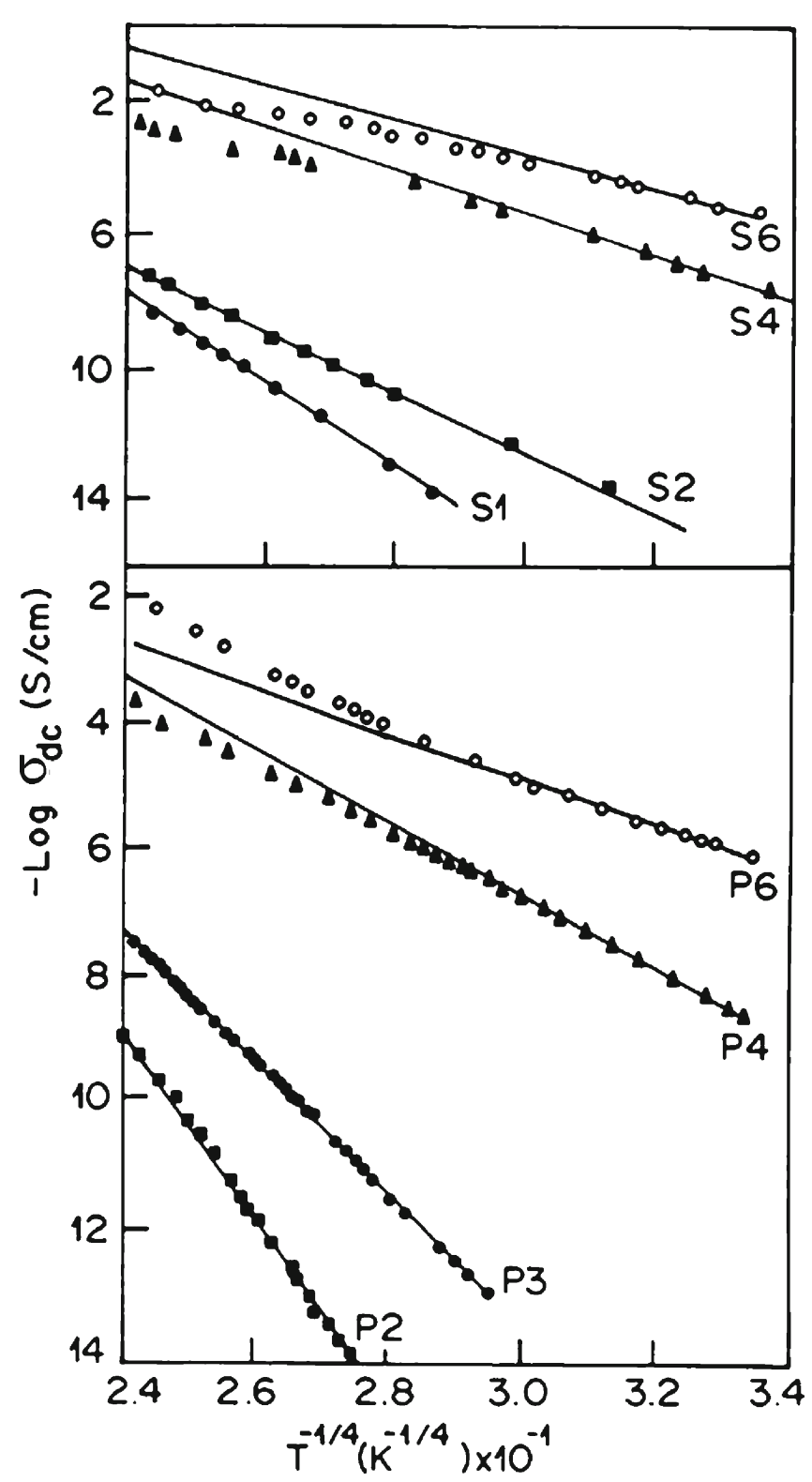

Figure 6. Variation of d.c. conductivity as a function of $T^{-1 / 4}$ for different $\mathrm{H}_{2} \mathrm{SO}_{4}$ and $\mathrm{H}_{3} \mathrm{PO}_{4}$ doped polyaniline samples.

in these figures from our previous work (Singh et al 1997) for the comparison of results at similar doping levels for these dopants. It is evident from figure 5, that for highly doped $S 4, S 6, P 4, P 6$ samples, the experimental conductivity data obey the $T^{-1 / 2}$ variation for full temperature range $77-300 \mathrm{~K}$ and for lightly doped samples, $S 1, S 2, P 2, P 3$, it is applicable in the low temperature region only. Further figure 6 shows that the conductivity data for lightly doped $S 1, S 2$, $P 2, P 3$ samples obey the $T^{-1 / 4}$ variation for whole temperature range and for highly doped $S 4, S 6, P 4, P 6$ samples, the experimental points follow the $T^{-1 / 4}$ in the low temperature region only. Therefore, these results reveal that in the case of $\mathrm{H}_{2} \mathrm{SO}_{4}$ and $\mathrm{H}_{3} \mathrm{PO}_{4}$ doped samples, 1D VRH mechanism is applicable for highly doped samples and 3D VRH mechanism is applicable for lightly doped samples i.e. electrical conductivity shows a change over from 3D VRH to 1D VRH for these multivalent ions at higher doping levels. However, in our earlier work (Singh et al 1998), Mott's 1D VRH mechanism was found applicable in polyaniline doped with $\mathrm{HCl}$ at all doping levels. From all these observations it may be concluded that hopping of the charge carriers in one dimension (1D) is taking place in the case of $\mathrm{HCl}$ doped polyaniline at all doping levels and for $\mathrm{H}_{2} \mathrm{SO}_{4}$ and $\mathrm{H}_{3} \mathrm{PO}_{4}$ doped polyaniline at high doping levels only. Further from these results it is evident that the value of Mott's VRH exponent ' $m$ ' depend upon the nature of the dopant ions used and its level of doping. However, more research data on different polymers are required to find the justified explanations for such experimental observations and to propose any theoretical model.

\section{Conclusions}

EPR studies have revealed that polarons and bipolarons are the main charge carriers formed during doping of the polyaniline with protonic acids used in the present investigation. Increase in doping level first causes increase in polaron concentration at low and moderate doping levels, whereas at very high doping levels, irrespective of any doping ions used, bipolarons are also formed. The mobility of these charge carriers increases at very high pace with increasing doping level due to increased inter-chain transport thereby electrical conductivity increases many folds. This is also confirmed by Dysonian line shape asymmetry parameter $A / B$ ratio of amplitudes having values higher than one at high doping levels. The concentration and effective mobility of the charge carriers formed as well as the d.c. electrical conductivity of the polyaniline have also been found to depend upon the nature of the dopants. The temperature dependence of d.c. conductivity supports the hopping conduction process and is well represented by Mott's variable range hopping (VRH) conduction model. The charge carriers show a changeover from 3D VRH to 1D VRH process for these multivalent ions at high doping levels. The electrical conductivity remains maximum in $\mathrm{HCl}$ doped polyaniline where 1D VRH has been followed for full doping range. The value of Mott's VRH exponent ' $m$ ' depends on the nature of dopant used as well as its doping level. These studies reveal that the inter-chain percolation causing increase in charge carriers mobility at high doping levels is mainly responsible for the observed electrical conductivity behaviour in polyaniline. However, more research work is required on different conducting polymers for proposing any quantitative model for estimating the effect of dopant ion on the electrical conductivity behaviour of any polymer.

\section{Acknowledgements}

Authors are grateful to the Director, National Physical Laboratory, New Delhi, for encouragement and support for this 
work. (SKG) and (RS) are thankful to the Council of Scientific and Industrial Research (CSIR), New Delhi, for emeritus scientist fellowship.

\section{References}

Alger R S 1968 Electron paramagnetic resonance-technique and applications (New York: Interscience Publishers) p. 494

Ayad M M, Salahuddin N A, Alghaysh M O and Issa R M 2010 Curr. Appl. Phys. 10235

Bredas J L and Street G B 1985 Acc. Chem. Res. 18309

Burroughes J H, Bradley D D C, Brown A R, Marks R N, Mackay K, Friend R H, Burns P L and Holmes A B 1990 Nature 347 539

Chiang J C and MacDiarmid A G 1986 Synth. Metals 13193

Chipara M, Aldica Gh, Hui D, Dimonie M, Lau K T, Georgescu L, Munteanu I and Marascoiu H 2004 J. Optoelectron. Adv. M. 6297

de Oliveria Jr J T and dos Santos M C 2000 Solid State Commun. $\mathbf{1 1 4} 49$

Dyson F J 1955 Phys. Rev. 98346

Elliott R J 1954 Phys. Rev. B96 266

Epstein A J 1997 Mater. Res. Soc. Bull. 2216

Feher G and Kip A F 1955 Phys. Rev. 98337

Formmer J E and Chance R R 1986 Encyclopedia of polymer science and engineering (ed.) H F Mark (New York: Wiley and Sons) 5 p.462

Houze E, Nechtschein M and Pron A 1997 Phys. Rev. B56 12263

Hutchison C A and Pastor R C 1951 Phys. Rev. 811282

Joshi J P and Bhatt S V 2004 J. Mag. Resonance 168284

Kang Y S, Lee H J, Namgoong J, Jung B and Lee H 1999 Polymer 402209

Krinichnyi V I, Tokarev S V, Roth H K, Schrodner M and Wessling B 2006 Synth. Metals 1561368

Kulkarni M V, Viswanath A K, Aiyer R C and Khanna P K 2005 J. Polym. Sci. B43 2161

Langer J J, Krzyminiewski R, Kruczynski Z, Gibinski T, Czajkowski I and Framski G 2001 Synth. Metals 122359
Lu W K, Elsenbaumer R L and Wessling B 1995 Synth. Metals 71 2163

Luthra V, Singh R and Mansingh A 2001 Synth. Metals 119291

Luthra V, Singh R, Gupta S K and Mansingh A 2003 Curr. Appl. Phys. 3219

Lux F 1994 Polymer 352915

MacDiarmid A G, Chiang J C, Richter A F and Epstein A J 1987 Synth. Metals 18285

Mizoguchi K, Nechtschein M, Travers J P and Menardo C 1989 Phys. Rev. Lett. 6366

Mott N F and Davis E A 1979 Electronic processes in non-crystalline materials (London: Oxford University Press) 2nd ed.

Punkka E, Rubner M F, Hettinger J D, Brooks J S and Hannahs S T 1991 Phys. Rev. B43 9076

Rouleau J F, Goyette J, Bose T K, Singh R and Tandon R P 1995 Phys. Rev. B52 4801

Scott J C, Pfluger P, Krounbi M T and Street G B 1983 Phys. Rev. B28 2140

Singh R, Narula A K, Tandon R P, Mansingh A and Chandra S 1996 J. Appl. Phys. 791476

Singh R, Arora V, Tandon R P, Chandra S, Kumar N and Mansingh A 1997 Polymer 384897

Singh R, Arora V, Tandon R P, Chandra S and Mansingh A 1998 J. Mater. Sci. 332067

Singh R, Arora V, Tandon R P, Mansingh A and Chandra S 1999 Synth. Metals 104137

Singh R, Kumar J, Singh R K, Chand S, Kumar V and Rastogi R C 2006a J. Appl. Phys. 10016106

Singh R K, Kumar J, Singh R, Kant R, Rastogi R C, Chand S and Kumar V 2006b New J. Phys. 8112

Stejskal J, Hlavata A, Holler P, Trchova M, Prokes J and Sapurina I 2004 Polym. Int. $\mathbf{5 3} 294$

Stephen C W and Frederic H I 1985 Microwave made simple: principles and applications (Chelsea, USA: United States Bookcrafters)

Wilamowski Z, Solnica M, Michaluk E, Havlicek M and Jantsch W 2011 Semicond. Sci. Technol. 26064009

Wu K H, Lai Y S, Shih C C, Wang G P and Yang C C 2008 Polym. Compos. 29902 